\title{
Uso de Plantas Medicinais por Famílias do Vale do Jequitinhonha, Minas Gerais, Brasil
}

\author{
Use of Medicinal Plants for Families from \\ Jequitinhonha Valley, Minas Gerais, Brazil
}

Recebido em: 14/12/2014

Aceito em: 17/03/2015
Maria Jesus Barreto CRUZ, Lays Fernanda Nunes DOURADO, Emerson Cotta BODEVAN, Lorena Ulhôa ARAÚJO, Cristiane Fernanda Fuzer GRAEL, Delba Fonseca SANTOS Universidade Federal dos Vales do Jequitinhonha e Mucuri - UFVJM. Campus JK. Rodovia MGT 367, Km 583, $n^{\circ}$ 5000. Alto da Jacuba. CEP 39100-000. Diamantina, MG, Brasil. E-mail: maria_enfermagem@yahoo.com.br

\section{ABSTRACT}

The aim of this study was to investigate the use of medicinal plants by the families living in the Jequitinhonha Valley, Minas Gerais. A descriptive study population-based survey was carried out by sampling of 555 households selected in a simple random way through cluster sampling of 137 census tracts. Of the participants, $73.51 \%$ reported the use of medicinal plants; and Mentha x piperita L., Mentha pulegium L. and Foeniculum vulgare Mill were the most cited species. Of those who affirmed to use herbs, $48 \%$ prepared them by decoction, $46.8 \%$ by infusion; $77 \%$ said that learned from their parents how to use and prepare the derivatives. It was observed that $90.7 \%$ interviewed said the plants were obtained from own backyards; $90.9 \%$ used to consume fresh plants and $94.4 \%$ said medicinal plants is safe. The use of medicinal plants is common in the studied cities. Thus, there is a need to conduct ethnobotanical research and training of health professionals to promote the rational use of medicinal plants.

Keywords: medicinal plant; rational use; ethnobotany

\section{RESUMO}

O objetivo do estudo foi investigar a utilização de plantas medicinais pelas famílias residentes no Vale do Jequitinhonha, Minas Gerais. Foi realizado um estudo descritivo tipo inquérito populacional domiciliar, amostra constituída por 555 domicílios selecionados de maneira aleatória simples por meio de amostragem por conglomerado de 137 setores censitários. Dos participantes, 73,51\% relataram a utilização de plantas medicinais, sendo Mentha x piperita L., Mentha pulegium L. e Foeniculum vulgare Mill as mais citadas. Dos que utilizaram plantas medicinais, 48\% preparavam por decocção, $46,8 \%$ por infusão, $77 \%$ aprenderam com os pais como utilizar e preparar. Foi observado que $90,7 \%$ obtiveram as plantas dos próprios quintais, $90,9 \%$ consumiam frescas e $94,4 \%$ achavam que não fazia mal. A utilização de plantas medicinais é comum nos municípios estudados. Assim, nota-se a necessidade de realização de pesquisa etnobotânica e capacitação dos profissionais de saúde para promover o uso racional de plantas medicinais.

Palavras chave: planta medicinal; uso racional; etnobotânica 


\section{INTRODUÇÃO}

Desde as mais antigas civilizações, o uso das plantas medicinais para diversas doenças representou, durante séculos, a única alternativa de cuidados e prevenção ao homem. Com o desenvolvimento da medicina convencional, essa utilização foi desvalorizada, uma vez que os profissionais de saúde consideram os medicamentos industrializados ou manipulados mais seguros. Apesar disso, é observado que o uso de plantas medicinais pode ser influenciado pela questão econômica, alto custo dos medicamentos, difícil acesso a consultas pelo Sistema Único de Saúde (SUS), dificuldade de locomoção de áreas rurais e tradição na utilização de recursos naturais (1).

Em 2002, a Organização Mundial da Saúde (OMS) reconheceu que $80 \%$ da população dos países em desenvolvimento utilizavam práticas tradicionais nos cuidados básicos de saúde e $85 \%$ usavam plantas medicinais ou preparações destas (2). Desde então, a OMS tem expressado sua posição a respeito da necessidade de valorizar a utilização de plantas medicinais no âmbito sanitário e na atenção básica à saúde (3).

Neste sentido, o Ministério da Saúde, com a intenção de implantar a utilização de práticas tradicionais e complementares no serviço público, aprovou, em 2006, diversas políticas importantes, tais quais: a Portaria ${ }^{\circ}$ 648, Política Nacional de Atenção Básica que incluiu as plantas medicinais no SUS (4); e a Portaria ${ }^{\circ} 971$ que aprovou a Política Nacional de Práticas Integrativas e Complementares (PNPIC), ampliou as opções terapêuticas, constituindo-se um norteador da utilização de plantas medicinais na saúde pública $(5,6)$. E, por fim, o Decreto ${ }^{\circ} 5.813$, Política Nacional de Plantas Medicinais e Fitoterápicos que estabeleceu diretrizes para o desenvolvimento de ações voltadas à garantia de acesso seguro e uso racional de plantas medicinais e fitoterápicos (7).

Como complementação dessas políticas foi elaborada a Relação Nacional de Plantas de Interesse ao SUS (RENISUS) em 2009, que contém uma lista de 71 espécies de plantas medicinais que apresentam potencial para gerar produtos de interesse ao SUS (8). Tais ações são imprescindíveis para aprimorar o acesso às plantas medicinais e aos fitoterápicos, favorecendo a promoção social e regional, o uso sustentável da biodiversidade e a manutenção do conhecimento e da cultura popular (9).

Considerando-se o valor das plantas medicinais, em 2010, a Secretaria de Estado de Saúde de Minas
Gerais lançou o programa Componente Verde da Rede Farmácia de Minas, como forma de ampliar as opções terapêuticas aos usuários do SUS. Este Programa constituiu-se como uma forma de acesso às plantas medicinais, enfatizando a necessidade de controle da qualidade de maneira equânime em toda a cadeia de produção até o consumidor final (10).

Por considerar a importância da utilização de plantas medicinais no cuidado a saúde pela população, Eldin \& Dunford (2001) afirmaram que estas ações são eficazes no atendimento primário, e complementam o tratamento, atendendo principalmente à população de menor renda (11). Neste sentido, em estudos realizados em unidades básicas de saúde, Badke e cols. (2011) e Feijó e cols. (2012) analisaram o uso das plantas medicinais no cuidado à saúde, e perceberam que o uso dessas plantas assume grande valor na vida destas pessoas (12, 13). Também afirmaram que o profissional da saúde, em especial o enfermeiro, deveria conhecer o contexto em que atuam, levando em consideração as plantas utilizadas pela comunidade, estudando sobre princípios ativos, contraindicações, nomes científicos e populares. Além disso, estes autores identificaram problemas em relação ao uso das plantas medicinais, como a forma de preparo inadequada, a procedência e o armazenamento impróprio, comprometendo a qualidade, propriedades funcionais e benefícios à saúde.

Neste contexto, a expansão da Estratégia Saúde da Família, contando com o Programa de Atenção Básica, denotou grande potencial para o desenvolvimento das ações com plantas medicinais e fitoterapia, ampliando o acesso da população aos benefícios dessa prática no SUS. Sobre automedicação, além do uso de medicamentos, o de plantas medicinais e remédios caseiros também são contemplados. Contudo há necessidade de cautela na escolha destas alternativas, em especial o uso de plantas, já que há a falta de estudos da efetividade e da segurança (14).

Com base nesses argumentos o presente estudo teve como objetivo investigar a utilização de plantas medicinais pelas famílias de crianças e adolescentes, residentes em áreas urbanas do Vale do Jequitinhonha, situada na região norte de Minas Gerais, por meio de um estudo epidemiológico de base populacional.

\section{MÉTODO}

Foi realizado um estudo descritivo e exploratório de corte transversal, tipo inquérito populacional domiciliar. As entrevistas foram realizadas por quatro entrevistadores treinados em estudo piloto para a validação 
da coleta, empregando um questionário adaptado e estruturado com perguntas abertas e fechadas (Figura 1) (15). A adaptação do questionário para o assunto plantas medicinais foi feita com base na oportunidade de inves- tigar o seu uso pela população estuda e também pelo fato deste tema ser de extrema importância diante dos aspectos culturais, sociais e econômicos do Vale do Jequitinhonha.

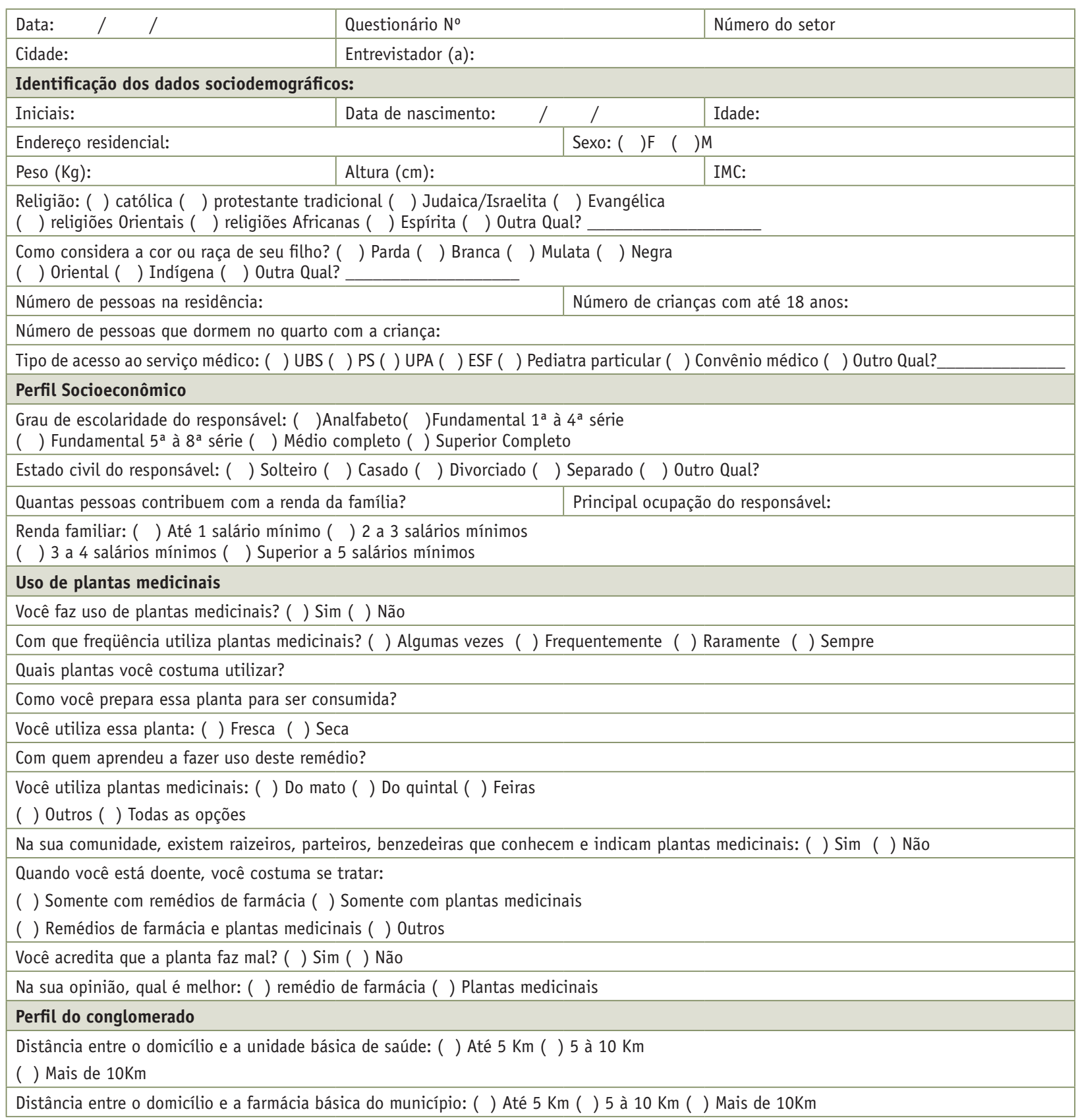

Figura 1: Uso de plantas medicinais nos municípios do Consórcio Intermunicipal de Saúde do Alto do Jequitinhonha - CISAJE

Os dados foram coletados na área urbana de 20 municípios do Vale do Jequitinhonha, Minas Gerais, Brasil, cadastrados no Consórcio Intermunicipal de Saúde do Alto Jequitinhonha (CISAJE), no período de 10 de abril a 20 de julho de 2013, a saber: Alvorada de Minas, Aricanduva, Capelinha, Carbonita, Congonhas do Norte,
Couto de Magalhães de Minas, Datas, Diamantina, Felício dos Santos, Gouveia, Itamarandiba, Leme do Prado, Minas Novas, Presidente Kubistchek, Santo Antônio do Itambé, São Gonçalo do Rio Preto, Senador Modestino Gonçalves, Serro, Turmalina, Veredinha. Os municípios estão distribuídos geograficamente conforme Figura 2. 


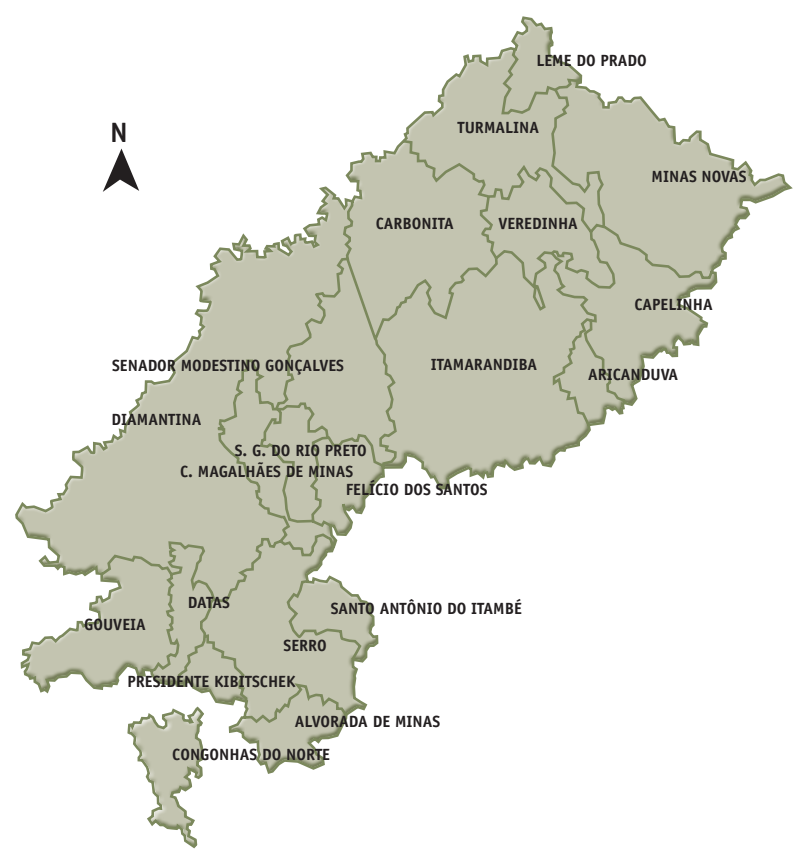

Figura 2: área do estudo etnobotânico: municípios do Consórcio Intermunicipal de Saúde do Alto do Jequitinhonha - CISAJE.

Adaptado de base de dados IBGE/2010.

Em relação ao Índice de Desenvolvimento Humano (IDH), cinco dos municípios possuem entre 0,558 a 0,$582 ; 14$ entre 0,616 a 0,682 e um com 0,716 , refletindo os indicadores de educação, habitação, saúde, trabalho, renda vulnerabilidade e longevidade, sendo que em Minas Gerais este índice é 0,731 e no Brasil é 0.744 (16).

Os domicílios foram selecionados de maneira aleatória simples por meio de amostragem em conglomerado, usando como unidade de referência 137 setores censitários urbanos para uma amostra de 672 domicílios definidos pelo Instituto Brasileiro de Geografia e Estatística (IBGE) (17). Foi feita a opção por sortear um maior número de setores, com a estimativa de não encontrar o número mínimo de indivíduos estipulados, principalmente em setores centrais (casa comerciais) e de bairros antigos com moradores idosos. Para cada setor sorteado, um mapa do IBGE foi impresso, permitindo aos pesquisadores encontrá-lo e locomover-se nele, seguindo uma sistemática pré-estabelecida para a seleção dos domicílios (17). Os critérios de inclusão foram famílias com crianças e adolescentes com idade menor ou igual a 14 anos, entrevista obrigatória com os responsáveis legais e ter hábito de consumo de plantas medicinais. Foram excluídos do trabalho os domicílios onde os responsáveis legais não estavam presentes no momento da entrevista ou se recusaram a concedê-la, quando o domicílio sorteado era estabelecimento comercial, e as residências nas quais não havia moradores com idade menor ou igual a 14 anos. Nos domicílios com mais de uma criança foi realizado apenas um questionário, sendo o indivíduo selecionado por sorteio, utilizando-se uma tabela de números aleatórios. Para determinar o número de participantes que comporiam a amostra foi utilizada a fórmula de estimação de proporção para população infinita:

$$
\mathrm{n}=\frac{\mathrm{p}(1-\mathrm{p}) z_{\gamma} / 2^{2}}{\varepsilon^{2}}
$$

onde:

- n: tamanho da amostra; pulação;

- p: proporção estimada de automedicação na po-

- zү/2: valor tabelado da distribuição normal padrão, correspondente a um nível confiança, $\gamma$, de 95\%);

$-\varepsilon$ : erro de estimação aceitável.

Foi considerado um erro de estimativa de 5\%, um nível de confiança de $95 \%$ e a suposição de desconhecimento da real prevalência de uso de plantas nos municípios $(\mathrm{p}=0,50)(18)$.

Foi estipulado o número calculado para a constituição da amostra de 672 entrevistas domiciliares para as zonas urbanas de cada cidade (erro aceitável de 5,0\% para uma amostra infinita). Para este cálculo, foram utilizados números do censo demográfico do IBGE do ano de 2010, que mostrou um total aproximado de 88.936 indivíduos com idade menor que 14 anos em 20 municípios do CISAJE, Diamantina, Minas Gerais (17).

A variável dependente foi utilização de plantas medicinais, e os participantes divididos em dois grupos de estudo: usam plantas medicinais e não usam plantas medicinais. Os dados sobre o uso de plantas somente foram coletados dos participantes que referiram fazer uso. Foram considerados três grupos de variáveis exploratórias: sócio-demográfica; utilização de serviço de saúde (público ou privado); existência de auxílio financeiro do governo (Programa Bolsa Família).

Como indicadores sócio-econômicos foram considerados: escolaridade do responsável; situação de moradia (se casa própria ou alugada); acesso a serviços de abastecimento de água; coleta de lixo e saneamento básico; renda familiar em salários mínimos vigentes à época. Foram coletadas as informações sobre as principais plantas medicinais consumidas pelas famílias, modo de preparo, frequência de uso, orientação do consumo e origem destas plantas. 
Para análise dos dados foi utilizado o Software Epi Info versão 7.0 (CDC/WHO, Atlanta, GE, EUA) e Microsoft Office Excel 2007, apresentados em figuras e tabelas, considerando os valores relativos e absolutos.

O estudo foi aprovado pelo Comitê de Ética em Pesquisa da Universidade Federal dos Vales do Jequitinhonha e Mucuri (UFVJM) (parecer 044/11); a população participante foi informada da finalidade do estudo e assinou o Termo de Consentimento Livre e Esclarecido (TCLE) pelos participantes da pesquisa.

\section{RESULTADOS E DISCUSSÃO}

O estudo incluiu uma amostra de 672 indivíduos, sendo que $117(17,4 \%)$ foram perdas e recusas, resultando em 555 entrevistas. Dessas, 73,5\% $(n=408)$ relataram utilização de plantas medicinais e $26,5 \%(n=147)$ não utilizavam plantas medicinais. Dentre os que relataram utilizar plantas, $35,8 \%$ possuíam o ensino médio, $24,35 \%$ eram analfabetos ou possuíam o ensino fundamental incompleto, $20,3 \%$ possuíam o ensino fundamental completo e apenas $19,6 \%$ concluíram o ensino superior.

Tabela 1: Análise das variáveis sociodemográficas em relação ao padrão de consumo de plantas medicinais - Municípios do Vale do Jequitinhonha (MG), Brasil, 2013

\begin{tabular}{|c|c|c|}
\hline Características da população & $\begin{array}{l}\text { Usa planta medicinal } \\
\qquad \begin{array}{c}n=408 \\
\%\end{array}\end{array}$ & $\begin{array}{l}\text { Não usa planta medicinal } \\
\qquad \begin{array}{c}\mathrm{N}=147 \\
\%\end{array}\end{array}$ \\
\hline \multicolumn{3}{|l|}{ Escolaridade do responsável } \\
\hline Analfabeto/Fundamental I ${ }^{\mathrm{a}}$ & 24,3 & 19,6 \\
\hline Fundamental II ${ }^{\mathrm{a}}$ & 20,3 & 19,1 \\
\hline Médio ${ }^{\mathrm{a}}$ & 35,8 & 42,2 \\
\hline Superior ${ }^{\mathrm{a}}$ & 19,6 & 19,1 \\
\hline \multicolumn{3}{|l|}{ Acesso ao serviço de saúde } \\
\hline Público & 73,1 & 77,5 \\
\hline Privado & 26,9 & 22,5 \\
\hline \multicolumn{3}{|l|}{ Renda (salários mínimos) b } \\
\hline$\leq 1$ & 40,9 & 41,5 \\
\hline $1-3$ & 40,7 & 42,2 \\
\hline $3-5$ & 10,3 & 8,8 \\
\hline$\geq 5$ & 8,1 & 7,5 \\
\hline \multicolumn{3}{|l|}{ Auxilio do governo (Programa Bolsa Família) } \\
\hline Possui & 36,0 & 34,7 \\
\hline Não possui & 64,0 & 65,3 \\
\hline
\end{tabular}

${ }^{a}$ Refere-se a escolaridade completa ou incompleta;- 'bSalário mínimo de R\$678,00.

Em relação ao acesso ao serviço de saúde $73,1 \%$ frequentavam o serviço público de saúde e $26,9 \%$ procuravam outro tipo de atendimento. Quando questionados quanto à existência de auxílio financeiro do governo $36 \%$ relataram receber (Tabela 1). Quanto à utilização de plantas medicinais, 90,9\% tinham o hábito de consumí-las frescas, $48 \%$ preparavam na forma de decocção. Dentre os entrevistados, $77 \%$ referiram que o aprendizado de como preparar e utilizar plantas medicinais foi oriundo de ensinamentos dos pais; $53,4 \%$ disseram não ter conhecimento da existência de pessoas na comunidade como benzedeiras, parteiras ou raizeiros que ensinassem ou indicavam o uso de plantas. Ao ficarem doentes, $59,6 \%$ informaram que utilizavam plantas medicinais e medicamentos de maneira conjunta, $70,6 \%$ acreditavam que o medicamento era melhor que a planta e $94,4 \%$ acreditavam que a planta medicinal não fazia mal (Tabela 2). 
Tabela 2: Análise das variáveis exploratórias em relação ao padrão de consumo de plantas medicinais - Municípios do Vale do Jequitinhonha (MG), Brasil, 2013

\begin{tabular}{|c|c|c|}
\hline Variáveis & Quantidade & $\%$ \\
\hline \multicolumn{3}{|l|}{ Forma como utiliza a planta } \\
\hline Seca & 37 & 9,1 \\
\hline Fresca & 371 & 90,9 \\
\hline \multicolumn{3}{|l|}{ Forma como prepara a planta } \\
\hline Infusão & 191 & 46,8 \\
\hline Decocção & 196 & 48,0 \\
\hline Outros & 21 & 5,2 \\
\hline \multicolumn{3}{|l|}{ Com quem aprendeu } \\
\hline Pais & 314 & 77,0 \\
\hline Parentes & 65 & 16,0 \\
\hline Conhecidos & 12 & 2,8 \\
\hline Escola/curso & 7 & 1,8 \\
\hline Não responderam & 10 & 2,4 \\
\hline \multicolumn{3}{|c|}{ Existem pessoas na cidade que ensinam a utilizar plantas medicinais } \\
\hline $\operatorname{Sim}$ & 190 & 46,6 \\
\hline Não & 218 & 53,4 \\
\hline \multicolumn{3}{|l|}{ Qual é melhor } \\
\hline Medicamento & 288 & 70,6 \\
\hline Planta medicinal & 120 & 29,4 \\
\hline \multicolumn{3}{|l|}{ Quando esta doente costuma se tratar } \\
\hline Com medicamentos e plantas medicinais & 243 & 59,6 \\
\hline Somente com plantas medicinais & 49 & 12,0 \\
\hline Somente com medicamentos & 112 & 27,4 \\
\hline Outra opção & 4 & 1,0 \\
\hline \multicolumn{3}{|l|}{ Acha que planta medicinal faz mal } \\
\hline Sim & 19 & 4,6 \\
\hline Não & 385 & 94,4 \\
\hline Não souberam responder & 4 & 1,0 \\
\hline
\end{tabular}

No que se refere ao grupo de plantas relatadas foram mencionadas 75 plantas e destas as 21 mais citadas (com nove citações ou mais) foram organizadas com seus respectivos nomes científico e popular, família e indicação científica ou etnobotânica (Tabela 3).

A utilização do poder curativo das plantas é um dos principais recursos terapêuticos utilizados pela humanidade desde as primeiras civilizações. Atualmente acredita-se que esse cuidado seja favorável à saúde humana, desde que realizados de maneira racional (12).
Neste estudo, foi verificado que $73,51 \%$ das famílias relataram utilização de plantas medicinais. Estes resultados corroboram com a afirmação de Almassy Junior (2004) em que o uso de plantas medicinais é valorizado deixando de ser costume de zona rural e chegando as cidades, como alternativa ao tratamento convencional, sendo um tratamento completar (23).

A partir do levantamento realizado, foi possível traçar o perfil socioeconômico e o acesso ao serviço de saúde da população estudada. 
Quanto à escolaridade, predominou os que cursaram o ensino médio (35,8\%), diferindo de estudo realizado por Souza e cols. (2013) em área urbana de Campina Grande, Paraíba (IDH 0,72, com renda de 1 a 2 salários mínimos), que evidenciou uma maior utilização de plantas medicinais pela população com ensino fundamental incompleto (49,9\%) (24). Mesmo diante do fato da amostra deste estudo ser restrita à área urbana dos 20 municípios, com um baixo IDH, 73,1\% referiram procurar atendimento público.

Quanto à renda familiar, 81,6\% dos entrevistados possuíam menos de três salários mínimos, sendo esta renda acrescida do Programa Bolsa Família do Governo Federal em 36\%; esses entrevistados referiram utilizar plantas medicinais. Os resultados corroboram aos encontrados por Arnous e cols.. (2005) em estudo realizado no município de Datas, MG, no qual foi verificado que $72,0 \%$ dos entrevistados apresentavam baixa renda, e que a utilização de plantas era um tratamento alternativo, uma opção de baixo custo (25).

Ao analisar o padrão de utilização das plantas medicinais como alternativa terapêutica para diferentes problemas de saúde, $75,5 \%$ relataram utilizar as plantas com frequência em seu cotidiano, resultado superior ao encontrado por Brasileiro e cols. (2008) que verificaram que $36,47 \%$ dos entrevistados utilizavam plantas medicinais com frequência, $55,47 \%$ utilizam raramente e apenas $8 \%$ não as utilizavam (26). No entanto, a divergência dos resultados obtidos neste trabalho se justifica pelo fato de que a população estudada ter sido aquela que utiliza o SUS e possui menor nível de escolaridade e renda, como observado por Costa \& Facchini (1997) (27). Neste contexto, a caracterização socioeconômica da amostra estudada sugere alta vulnerabilidade social dos municípios estudados, refletida principalmente pela baixa escolaridade dos responsáveis e pela baixa renda das famílias.

A decocção e a infusão são formas de preparo de chás. Os chás, normalmente são feitos a partir de plantas aromáticas, que devem ser empregadas na forma fresca, para se evitar perdas dos óleos essenciais. Os óleos essenciais são constituídos principalmente por misturas de substâncias terpênicas e compostos fenólicos (como os fenilpropanoides), que apresentam variadas atividades biológicas e farmacológicas $(22,28)$. O processo de preparo de chás por decocção é mais indicado para partes duras do vegetal (raízes, caules, cascas, frutos secos, sementes), enquanto a infusão é mais empregada quando se utilizam folhas, flores ou estruturas de tecidos mais delicados (22).

O material vegetal utilizado fresco, sem secagem, garante maior teor de princípios ativos aromáticos pre- sentes. Por outro lado, o uso de aquecimento para o preparo de decocção e de infusão, apesar de favorecer a extração do óleo a partir do tecido vegetal, resulta em perdas de óleo por volatilização. Além disso, em processos extrativos, como a decocção e a infusão, que envolvem o aumento de temperatura e a presença da água, podem ocorrer oxidações, rearranjos, hidrólises, racemizações e isomerizações de constituintes do óleo essencial, resultando na presença de artefatos (28). No entanto, muitas plantas aromáticas tem seu uso popular consagrado na forma de decocção e de infusão preparados por infusão ou decocção; isso pode acontecer porque, apesar das perdas e alterações de constituintes no processo de preparo, os decoctoses os infusos, apresentam propriedades curativas ou paliativas.

Neste estudo, foram identificadas duas formas predominantes de preparo da planta: $48 \%$ apontaram a preparação na forma de decocção, $46,8 \%$, na forma de infusão. Uma pequena parcela $(5,2 \%)$ utilizou outros métodos como sumo ou maceração. Em estudo realizado por Arnous e cols. (2005), o processo citado pela maior parte dos entrevistados foi a decocção (25).

Conforme os dados levantados no presente estudo, o predomínio das informações sobre a utilização de plantas medicinais foi o saber familiar (77\%), sendo que resultados semelhantes foram encontrados por Santos e cols. (2009) (29) e por Marinho e cols. (2011), onde 85\% dos entrevistados afirmaram que o aprendizado sobre o uso das plantas medicinais foi adquirido por intermédio dos pais (30). Sendo assim, a medicina popular é um saber fundamentado na transmissão oral de conhecimentos das famílias (24), embasada em grande parte na cultura dos povos (31).

Segundo Sacramento (2001), no Brasil a fitoterapia tem eficácia e legitimidade reconhecidas devido às profundas raízes na consciência popular (32), sendo de fundamental importância a manutenção de tal saber. Entretanto, deve ser ressaltado que as pessoas detentoras deste conhecimento são aquelas mais idosas e com mais baixo nível de escolaridade, o que contrapõe com o baixo interesse na fitoterapia pelos mais jovens, como melhor nível de escolaridade, como se observam em alguns estudos $(33,34)$.

Destaca-se também, que entre as famílias que relataram utilizar plantas medicinais, 94,4\% achavam que este uso não faz mal, que são destituídas de efeito adverso e não apresentam contra indicações; e 59,6\% relataram que, estando doentes, usam as plantas em associação com medicamentos. O uso predominante desta associação, também foi encontrado em um estudo realizado por Rosa e cols. (2012), que comprovaram o uso de 
plantas medicinais concomitante a medicamentos para 93,1\% dos participantes (31). Deve ser ressaltado que a interação entre medicamentos e plantas medicinais pode diminuir ou potencializar os efeitos de ambos (35), uma vez que as plantas medicinais são constituídas de misturas complexas de muitos compostos químicos, que podem ser responsáveis pelas suas ações polivalentes (36). Contudo, em muitos casos, os constituintes químicos responsáveis pelas atividades farmacológicas das plantas medicinais são desconhecidos e a complexidade dos constituintes presentes aumenta a possibilidade de ocorrer interações quando fármacos são utilizados concomitantemente (37).

Contradizer esse costume é difícil, pois os relatos científicos de intoxicações e efeitos colaterais relacionados com o uso de plantas medicinais são informações pouco acessíveis aos usuários da rede de saúde pública, pois, na maioria, são de baixa escolaridade. Aliado a isso, deve ser considerado que essas informações não são de amplo conhecimento entre os profissionais do serviço público de saúde (38).

Foi observado ainda que $90,7 \%$ das famílias obtiveram as plantas medicinais dos próprios quintais e 90,9\% tinham o hábito de consumí-las frescas. Resultado semelhante foi encontrado por Brasileiro e cols. (2008) onde 547 pessoas responderam que obtinham as plantas em plantações próprias (26). Estes dados do presente trabalho também coincidem com os estudos de Rosa e cols. (2012) e Pilla e cols. (2006), que relataram ser o quintal das casas o local mais citado pelos indivíduos como fonte de obtenção das espécies vegetais, o que reforça o fato de que a utilização de plantas medicinais é facilitada pela obtenção das mesmas, pois são cultivadas pelos próprios usuários e preparadas ainda frescas $(31,39)$. Assim, a complementação do conhecimento popular e científico sobre a produção e o uso de plantas medicinais é fundamental para sua segurança e eficácia (26).

No que se refere ao grupo de plantas relatadas neste estudo foram mencionadas 75 plantas e destas, as 21 mais citadas (com nove citações ou mais) foram organizadas com seus respectivos nomes científico e popular, família e indicação científica ou etnobotânica. Em trabalhos desta natureza é importante a identificação botânica da planta, pois ocorre a designação do mesmo nome popular para diferentes espécies vegetais dificultando a identificação (40). As plantas mais citadas são bastante comuns e muito conhecidas e sua identificação foi realizada por comparação com a literatura (19-22) ou em herbários virtuais (41).

Das 21 espécies mais citadas, a maioria (dez) pertence à família Lamiaceae, reconhecida pela marcante presença de óleos essenciais, sendo por isso consideradas plantas aromáticas (28). Dentre as espécies mais citadas pelos entrevistados, Cymbopogon citratus (DC.) Stapf.(capim-cidreira), Foeniculum vulgare Mill. (funcho), Gossypium barbadense L. e G. hirsutum L. (algodão) Mentha pulegium L.(poejo) e Ocimum gratissimum L. (alfavaca) são subespontâneas e não endêmicas da flora brasileira, de acordo com Forzza e cols. (2010) (42). As plantas subespontâneas são aquelas que, oriundas de outras regiões do planeta, se instalam em um país sem a interferência deliberada do homem. Somente as espécies Achyrocline satureioides (Lam) DC. (macela ou macelica), Mikania sp (guaco) e Phyllanthus sp (quebra-pedra) são nativas do Brasil e não endêmicas. As demais espécies citadas pelos entrevistados, inclusive a mais citada, hortelã - Mentha x piperita L. (com 277 citações) -, são espécies exóticas e adaptadas ao cultivo no Brasil. Muitas plantas nativas do Cerrado (bioma predominante na região estudada) podem apresentar porte maior, de cultivo difícil em quintais, e por isso existindo uma forte tradição no uso das plantas aromáticas e de pequeno porte, que são cultiváveis facilmente. Sendo assim, explica-se porque a maioria das plantas citadas pelos entrevistados são exóticas. Além disso, essas plantas exóticas são tradicionalmente empregadas na região, e um estudo anterior realizado no município vizinho de Datas (MG) indicou várias dessas espécies como cultivadas nos quintais e utilizadas para fins medicinais (25).

A partir dos resultados obtidos, foi possível verificar que a utilização de plantas medicinais é bastante comum nos municípios estudados, principalmente quando relacionado às características sociodemográficas locais como a baixa renda, escolaridade e acesso ao serviço público de saúde. Tais atitudes denotam a utilização de plantas como uma alternativa complementar aos tratamentos de saúde existentes, embora os entrevistados, em sua maioria, afirmem que o medicamento é melhor do que a utilização de plantas ou acabam utilizando-os concomitantemente.

Diante do expressivo número de plantas relatadas, pode ser notada a necessidade de se realizar pesquisa etnobotânica na região, a fim de promover a valorização do conhecimento tradicional, especialmente das plantas nativas, e a identificação dos principais motivos de uso dessas plantas pela população. Além disso, para se estabelecer um programa de plantas medicinais e fitoterápicos no serviço público regional de saúde é importante conhecer as plantas mais empregadas e aceitas pelos usuários do SUS. Essas plantas, sendo eficazes e seguras para uso humano, poderão fazer parte de programas para cultivo e processamento e sua distribuição para a população. Nesse caso, faz-se necessário desenvolvimento de 
cursos de capacitação para os profissionais da saúde que deverão orientar a população quanto ao uso das plantas medicinais e também dos fitoterápicos. Além disso, é muito importante a determinação da eficácia e segurança das plantas para o uso pelo serviço público, pois há, por exemplo, entre as plantas mais citadas nesta pesquisa, o poejo (com 144 citações), que em doses elevadas pode ser abortivo e hepatóxico, de acordo com outros estudos publicados (22).

Neste contexto, pode ser observada a necessidade da inclusão do conhecimento de terapias alternativas, como a fitoterapia, no cotidiano de profissionais da saúde. Essa inclusão abre maiores possibilidades para que estes profissionais realizem as atividades em caráter multidisciplinar, buscando melhorar a saúde e a qualidade de vida da população, proporcionando novas opções de tratamento para as patologias (31).

\section{CONCLUSÃO}

Diante dos resultados obtidos neste estudo, foi observado que a utilização de plantas medicinais é expressiva, mesmo em áreas urbanas. Aproximadamente $74 \%$ dos entrevistados utilizavam plantas medicinais como alternativa de tratamento de baixo custo. $\mathrm{O}$ grande número de plantas citadas reafirma a importância de realizar pesquisa etnobotânica na região, que promova a valorização do conhecimento tradicional (em especial das plantas nativas) e identifique os principais motivos de uso dessas pela população. Sendo assim, o estudo sugere verificar as possibilidades de investigar as potencialidades da população quanto ao uso de plantas medicinais e consequentemente contribuir com a implantação das Políticas Nacionais de Plantas Medicinais e Fitoterápicos e de Práticas Integrativas e Complementares.

Também foi constatada a necessidade de domínio desse saber pelos profissionais de saúde, uma vez que a utilização de plantas medicinais deve ser estudada e aperfeiçoada para ser realizada com segurança e eficácia. É necessário integrar o saber popular ao saber científico, valorizar a cultura local e promover de maneira integral a oferta de cuidados.

\section{AGRADECIMENTOS}

Este estudo foi financiado pela Fundação de Amparo a Pesquisa do Estado de Minas Gerais (FAPEMIG-Processo ${ }^{\circ}$ CDS-APQ-02522-11).

\section{REFERÊNCIAS}

1. Battisti C, Garlet TMB, Essi L, Horbach RK, Andrade A, Badke MR. Plantas medicinais utilizadas no município de Palmeira das Missões, RS, Brasil. R Bras Bioci. 2013. 11(3): 338-348.

2. OMS. Organização Mundial de Saúde. Estrategia de la OMS sobre medicina tradicional 2002-2005. 2002. Genebra. 67 p.

3. Rosa C, Câmara SG, Béria JU. Representações e intenção de uso da fitoterapia na atenção básica à saúde. Ciênc Saúde Colet. 2011. 16(1): 311 - 318 .

4. BRASIL. Ministério da Saúde. Portaria $n^{\circ} 648$, de 28 de março de 2006. Aprova a Política Nacional de Atenção Básica, estabelecendo a revisão de diretrizes e normas para a organização da Atenção Básica para o Programa Saúde da Família (PSF) e o Programa Agentes Comunitários de Saúde (PACS). Diário Oficial da União [da República Federativa do Brasil], Brasília. 2006a. seção 1, n. 61, p. 71.

5. BRASIL. Ministério da Saúde. Portaria $n^{\circ} 971$ de 3 de maio de 2006. Aprova a Política Nacional de Práticas Integrativas e Complementares (PNPIC) no SUS. Diário Oficial da República Federativa do Brasil. 2006c. Brasília, DF.

6. BRASIL. Ministério da Saúde. A fitoterapia no SUS e o Programa de Pesquisa de Plantas Medicinais da Central de Medicamentos. Brasília: Ministério da Saúde. 2006d. $148 \mathrm{p}$.
7. BRASIL. Ministério da Saúde. Secretaria de Ciência, Tecnologia e Insumos Estratégicos. Departamento de Assistência Farmacêutica. Política Nacional de Plantas Medicinais e Fitoterápicos/ Ministério da Saúde, Secretaria de Ciência, Tecnologia e Insumos Estratégicos, Departamento de Assistência Farmacêutica. Brasília: Ministério da Saúde. 2006b. 60p.

8. BRASIL. Ministério da Saúde. Direção de Administração e Finanças. Secretaria de Ciência, Tecnologia e Insumos Estratégicos. RENISUS - Relação Nacional de Plantas Medicinais de Interesse ao SUS. 2009b. 1p. Disponível em: <http://portal.saude.gov.br/ portal/arquivos/pdf/ RENISUS.pdf $>$. Acesso em: 05/06/14.

9. BRASIL. Ministério da Saúde. Secretaria de Atenção à Saúde. Departamento de Atenção Básica. Práticas integrativas e complementares: plantas medicinais e fitoterapia na Atenção Básica/Ministério da Saúde. Secretaria de Atenção à Saúde. Departamento de Atenção Básica. - Brasília: Ministério da Saúde, 2012. 156 p.: il. - (Série A. Normas e Manuais Técnicos) (Cadernos de Atenção Básica; n. 31).

10. Sant'ana JMB, Pepe VLE, Osorio-de-Castro CGS, Ventura $\mathrm{M}$. Essencialidade e assistência farmacêutica: considerações sobre o acesso a medicamentos mediante ações judiciais no Brasil. Rev Panam Salud Publica. 2011. 29(2): 138-44. 
11. Eldin S, Dunford A. Fitoterapia na atenção primária a saúde. São Paulo: Manole. 2001.163 p.

12. Badke MR, Budó MLD, Silva FM, Ressel LB. Plantas medicinais: o saber sustentado na prática do cotidiano popular. Esc Anna Nery. 2011. 15 (1): 132-139.

13. Feijó AM, Bueno MEN, Ceolin T, Linck CL, Schwartz E, Lange C, Meincke SMK, Heck RM, Barbieri RL, Heiden G. Plantas medicinais utilizadas por idosos com diagnóstico de Diabetes mellitus no tratamento dos sintomas da doença. Rev Bras Plantas Med. 2012. 14(1): 50-56.

14. Gentil LB, Robles AC, Grosseman S. Use of complementary therapies by mothers in their children: study at an university hospital. Ciênc Saúde Coletiva. 2010. 15(1): 1293-1299.

15. Tourinho FSV, Bucaretchi F, Stephan C, Cordeiro, R. Farmácias domiciliares e sua relação com a automedicação em crianças e adolescentes. J Pediatr. 2008; 84: 416 - 422.

16. IPEA. Instituto de Pesquisa Econômica Aplicada [Internet]. Atlas do Desenvolvimento Humano do Brasil 2013. Municípios e Faixa de Desenvolvimento Humano. 2013. Disponível em: http://www.atlasbrasil.org.br/2013/. Acesso em: 18/01/14.

17. IBGE. Instituto Brasileiro de Geografia e Estatística. População e Domicílios - Censo 2010 com Divisão Territorial 2001, Minas Gerais. 2010. [site na Internet] 2010. Diponível em: http://downloads.ibge.gov.br/downloads geociencias.htm. Acessado em: 03/05/2013.

18. Schwambach KH. Utilização de plantas medicinais e medicamentos no autocuidado no município de Teutônia, RS. [Dissertação]. Porto Alegre: Faculdade de Farmácia, Programa de Pós-Graduação em Ciências Farmacêuticas, UFRGS. 2007.

19. Alonso JR. Fitomedicina - curso para profissionais da área da saúde. São Paulo: Pharmabooks. 2008.

20. Barros FMC, Pereira KN, Zanetti GD, Heinzmann BM. Plantas de uso medicinal no município de São Luiz Gonzaga, RS, Brasil. Lat Am J Pharm. 2007. 26(5): 652-662.

21. Brandão MGL. Plantas úteis de Minas Gerais - na obra dos naturalistas. Belo Horizonte: Código Comunicação. 2010.

22. Lorenzi H, Matos FJA. Plantas medicinais no Brasil - nativas e exóticas. Nova Odessa, SP: Instituto Plantarum. 2002.

23. Almassy Júnior AA. Análise das características etnobotânicas e etnofarmacológicas de plantas medicinais na comunidade de Lavras Novas, Ouro Preto-MG. 2004. 130p. Tese (Doutorado em Fitotecnia) - Universidade Federal de Viçosa, Viçosa. 2004.

24. Souza CMP, Brandão DO, Silva MSP, Palmeira AC, Simões MOS, Medeiros ACD. Utilização de plantas medicinais com atividade antimicrobiana por usuários do serviço público de saúde em Campina Grande - Paraíba. Rev Bras Plantas Med. 2013. 15 (2): 188-193.

25. Arnous AH, Santos AS, Beinner RPC. Plantas medicinais de uso caseiro - conhecimento popular e interesse por cultivo comunitário. Rev Espaço para Saúde. 2005.6(2): 1-6.

26. Brasileiro BG, Pizziolo VR, Matos DS, Germano AM, Jama CMA. Plantas medicinais utilizadas pela população atendida no Programa de Saúde da Família, Governador Valadares, MG, Brasil. Rev Bras Cienc Farm. 2008. 44(4): 629-636.
27. Costa JSD, Facchini LA. Utilização de serviços ambulatoriais em Pelotas: onde a população consulta e com que frequência. Rev Saúde Pública. 1997. 31: 360-369.

28. Simões CMO, Schenkel EP, Gosmann G, Mello JCP, Mentz LA, Petrovick PR. Farmacognosia - da planta ao medicamento. 6. ed. Porto Alegre/Florianópolis: UFRGS/ UFSC. 2007.

29. Santos EB, Dantas GS, Santo SHB, Diniz MFFM, Sampaio FC. Estudo etnobotânico de plantas medicinais para problemas bucais no município de João Pessoa, Brasil. Rev Bras Farmacogn. 2009. 19(1B): 321-324./27.

30. Marinho MGV, Silva CC, Andrade LHC. Levantamento etnobotânico de plantas medicinais em área de caatinga no município de São José de Espinharas, Paraíba, Brasil. Rev Bras Plantas Med. 2011. 13(2): 170-182.

31. Rosa RL, Barcelos ALV, Bampi G. Investigação do uso de plantas medicinais no tratamento de indivíduos com diabetes melito na cidade de Herval D' Oeste - SC. Rev Bras Plantas Med. 2012. 14(2): 306-310.

32. Sacramento HT. Legislação para produção, comercialização e uso de plantas medicinais. In: Jornada Paulista de Plantas Medicinais, 5.; 2001. Botucatu. Anais. Botucatu: UNESP, p.33. 2001.

33. Brandão MGL, Zanetti NNS, Oliveira GR, Silva AKS, Goulart LO, Oliveira MA, Grael CFF, Santos ACP. Plantas medicinais da Estrada Real MG-Biota. 2008.1(4): 4-15.

34. Medeiros MFT, Fonseca VS, Andreata RHP. Plantas medicinais e seus usos pelos sitiantes da Reserva Rio das Pedras, Mangaratiba, RJ, Brasil. Acta Bot Bras. 2004.18:391-399.

35. Alexandre RF, Bagatini F, Simões CMO. Interação entre fármacos e medicamentos fitoterápicos a base de ginko ou ginseng. Rev Bras Farmacogn. 2008. 18(1): 117- 26.

36. Williamson EM. 2005. Interactions between herbaland conventional medicines. Expert Opin Drug Saf. 2005. 4(2): 355-378

37. Fugh-Bernman A, Ernst E. Herb-drug interactions: review and assessment of report reability. Br J Clin Pharmacol. 2001. 52(5): 587-595.

38. Silveira PF, Bandeira MAM, Arrais PSD. Farmacovigilância e reações adversas às plantas medicinais e fitoterápicos: uma realidade. Rev Bras Farmacogn. 2008. 18(4): 618-626.

39. Pilla MAC, Amorozo MC, Furlan A. Obtenção e uso das plantas medicinais no distrito de Martim Francisco, município de Mogi-Mirim, SP, Brasil. Acta Bot Bras. 2006. 20(4): 789-802.

40. Penso G. The role of WHO in the selection and characterization of medicinal plants (vegetables drugs). J Ethnopharmacol.1980. 2(2): 183-188.

41. INCT-HVFF. Instituto Nacional de Ciência e Tecnologia Herbário Virtual da Flora e dos Fungos. [Acesso em 20/01/2014]. Disponível em: http://inct.florabrasil.net/. Reflora - Herbário Virtual. Disponível em: http://www. herbariovirtualreflora.jbrj.gov.br/jabot/herbarioVirtual/

42. Forzza RC. et al. Catálogo de plantas e fungos do Brasil. Instituto de Pesquisas Jardim Botânico do Rio de Janeiro. 2010 . 